\title{
Chiral Born-Infeld Theory: topological spherically symmetrical solitons
}

\author{
O.V. Pavlovskiu * \\ Bogoliubov Institute for Theoretical Problems of Microphysics, \\ Lomonosov Moscow State University \\ Moscow, 119992, Russian Federation.
}

\begin{abstract}
Finite-energy topological spherically symmetrical solutions of Chiral Born-Infeld theory are studied. Properties of these solution are obtained, and a possible physical interpretation is also given.
\end{abstract}

PACS number(s): 12.39.Dc, 12.39.Fe, 05.45.Yv, 11.27.+d, 11.30.Qc

\section{Introduction}

The concept of the baryon as a chiral soliton has a long history. The idea of a unified theory for baryons and mesons that is formulated in terms of chiral field was only proposed for the first time by Skyrme [1]. The well-known Skyrme Model is used for analyzing static properties of barions, for describing nucleon-nucleon forces, meson-nucleon scattering and for many other low-energy aspects of baryons and mesons physics.

In the seventies 't Hooft and Witten [2] have shown that, in the limit $N_{c} \rightarrow \infty$, QCD can be reformulated in terms of effective meson theory like Skyrme Model and that chiral solitons of such theory reproduce the static properties of real baryons. These facts attracted special attention to the theories with chiral solitons.

Effective meson field theory can be formulated in terms of chiral field $U=\exp \left(i \frac{\vec{\phi}_{\pi} \vec{\tau}}{f_{\pi}}\right)$ where the vector field $\vec{\phi}_{\pi}$ is associated with $\pi$ - mesons and $f_{\pi}=93 \mathrm{MeV}$ is the pion decay constant. The simplest chiral invariant prototype lagrangian for mesons field $\vec{\phi}_{\pi}$ is given by

$$
\mathcal{L}_{p r}=-\frac{f_{\pi}^{2}}{4} \operatorname{Tr} L_{\mu} L^{\mu}
$$

where $L_{\mu}=U^{+} \partial_{\mu} U$ is a Cartan left-invariant form (see reviews [3]). However using the scale transformation analogous to the Derrick Theorem 四 one can find that theory with the lagrangian (11) has no finite energy static solutions and all models with chiral soliton appear to be various modifications of the prototype lagrangian. For example, the skyrmeon

*e-mail address: ovp@goa.bog.msu.su 
(chiral soliton ofthe Skyrme Model) exists due to the including of the Skyrme-part $\Delta \mathcal{L}_{S k}=$ $\operatorname{Tr}\left[L_{\mu}, L_{\nu}\right]^{2} / e^{2}$ in lagrangian of this model. This theory has a set of topological static solutions and one can classify these solutions by the value of the topological (baryon) charge

$$
B=\frac{1}{24 \pi^{2}} \int d^{3} \epsilon_{i j k} \operatorname{Tr}\left(L_{i} L_{j} L_{k}\right) .
$$

In this model such solutions are associated with baryon states with different baryon charges, and soliton with $B=1$ (skyrmeon) is treated as a nucleon. Such kind of solutions and quantum fluctuations about them is well studied now (see [3]) and results of such analysis are in agreement with the experiment data with accuracy of 30-40 percents for baryonic masses and another static properties of baryons.

But there are many other ways for stabilization of chiral soliton, because from the methodological point of view, arising of the Skyrme part in the lagrangian of effective meson field theory is an "ad hoc" procedure, and there are no real physical bases for this procedure. Of course, one can treat this part in the effective lagrangian as a leading high derivative expansion of effective chiral meson action (see review [0] ), but this approach leads to numerous questions so far. Two most important ones concern the physical nature of the scale parameter (e) in this theory and the influence of another terms of expansion on existence and stability of chiral solitons.

Another possible way of stabilizing of chiral solitons was suggested in [6]. In these works the role of the vector $(\rho)$ and pseudo-scalar $(\omega)$ mesons in chiral soliton physics is discussed. Procedure of $\omega$-stabilization leads to forming of topological soliton solution. Many other procedures of stabilization were proposed so far. One such approach that allows to get over the difficulty connected with scale instability of chiral solitons in $3+1$ dimension was proposed in [7]. Indeed, prototype lagrangian (11) has well-known stable static topological solitons in $1+1$ dimension [9]. This situation stems from the fact of dimensionless of the chiral action in $1+1$ dimension. By analogy with $1+1$ dimension case, in [7] the model with the lagrangian

$$
\mathcal{L}_{3 / 2}=-\frac{f_{\pi}^{2}}{4}\left(\operatorname{Tr} L_{\mu} L^{\mu}\right)^{3 / 2},
$$

was considered. The authors of [8] name this model Born-Infeld-Skyrme Model because of the half-integer power in expression (3).

In [10] another type of a Born-Infeld-Skyrme Model with the lagrangian

$$
\mathcal{L}_{S k B I}=-\frac{f_{\pi}^{2}}{4} \operatorname{Tr} L_{\mu} L^{\mu}+\beta^{2}\left(\sqrt{1+\frac{1}{16 e^{2} \beta^{2}} \operatorname{Tr}\left[L_{\mu}, L_{\nu}\right]^{2}}-1\right)
$$

was proposed. This model satisfies the basic requirements for models of Born-Infeld type and have stable soliton solution, but the Born-Infeld part in the lagrangian (勾) plays the role of Skyrme-term in the lagrangian (3i) and this model is one possible modification of Skyrme's procedure.

The consistent effective low-energy meson's theory construction is a very complicated task that is closely connected to the quark's confinement problem and to the problem of spontaneous breaking of chiral symmetry. It is desirable that such theory should be Lorentz and chiral invariant and should have a set of finite energy stable solutions. In the case of electro-magnetic fields such kind of an effective low-energy theory is the well-known BornInfeld model [11]. In this work we study the direct analogue of the Born-Infeld action for chiral fields. By construction our model has no singular solutions and looks very attractive as a possible effective action for mesons fields. This model has a set of stable topological solitons. 
These solutions can be treated as baryons states in our model. The physical motivation of our model is beyond the scope of this article.

In Section 2, we discuss the problem of constructing of a Born-Infeld (BI) action for the chiral field (ChBI model). The lagrangian and equation of motion are presented. The spherically symmetrical configurations of chiral field are considered and topological spherically symmetrical solitons are studied. In Section 3 we summarise the results of this paper.

\section{The Born-Infeld theory for Chiral Fields}

In the well-known paper [11], Born and Infeld proposed a non-linear covariant action for electro-magnetic fields with very attractive features. Firstly, in the framework of BI theory the problem of singular self-energy of electron can be solved. In this theory the electron is a stable finite energy solution of the BI field equation with electric charge. Second, the BI action has a scale parameter $\beta$. Using expansion by this parameter one reduces the BI action to the usual Maxwell form in the low-energy limit.

We want to perform a very similar procedure with the chiral prototype lagrangian (1). Like in the case of BI action, our chiral model must have a set of finite energy solutions with integer values of charge (topological or baryon), and in the low-energy limit such a theory must reproduce the prototype lagrangian (1). The model must be Lorentz and chiral invariant. Finally, the form of such theory follows from the analogy with the action of a relativistic particle, the BI action for EM and YM field. This form has geometrical nature and corresponds to a number of analogies between chiral dynamics, gauge theory and general relativity [12].

Arguing as above, let us consider a theory with lagrangian

$$
\mathcal{L}_{C h B I}=-f_{\pi}^{2} \operatorname{Tr} \beta^{2}\left(1-\sqrt{1-\frac{1}{2 \beta^{2}} L_{\mu} L^{\mu}}\right) \sim-\frac{f_{\pi}^{2}}{4} \operatorname{Tr} L_{\mu} L^{\mu},
$$

where $\beta$ is a mass dimensional scale parameter of our model. It is easily shown that the expansion of the lagrangian (5) gives us the prototype theory as the leading order theory by parameter $\beta$. Our theory contains only second-order derivative terms. Thus the dynamics of this field theory can be studied in detail.

Now we consider the spherically symmetrical field configuration

$$
U=e^{i F(r)(\vec{n} \vec{\tau})}, \quad \vec{n}=\vec{r} /|r|
$$

The energy of such field configuration is the functional

$$
E^{\beta}[F]=8 \pi f_{\pi}^{2} \beta^{2} \int_{0}^{\infty}(1-R) r^{2} d r
$$

where

$$
R=\sqrt{1-\frac{1}{\beta^{2}}\left(\frac{F^{\prime 2}}{2}+\frac{\sin ^{2} F}{r^{2}}\right)}
$$

Using the variation principle, we get the equation of motion

$$
\left(r^{2} \frac{F^{\prime}}{R}\right)^{\prime}=\frac{\sin 2 F}{R}
$$




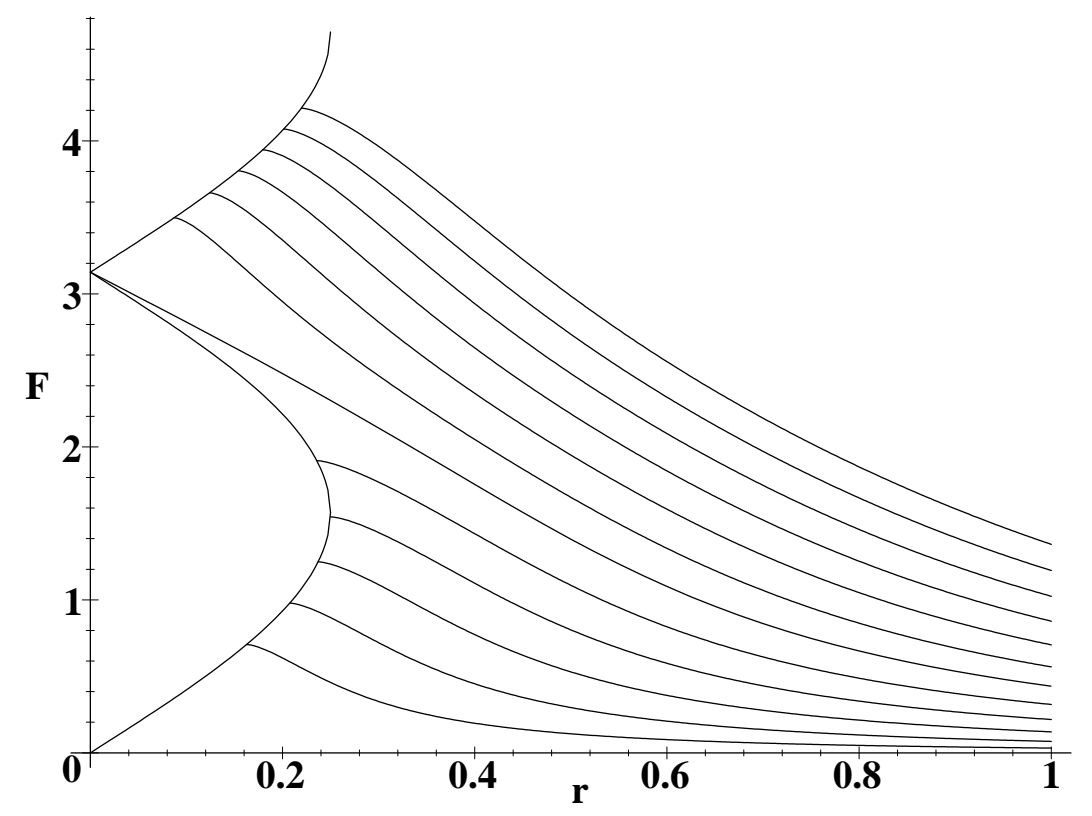

Figure 1: Solutions of equation (9) which have the asymptotics $(12)(b>0)$ at infinity. Horizontal axis: $\mathrm{r}$ (in fm).

and for amplitude $F(r)$ we obtain

$$
\begin{gathered}
\left(r^{2}-\frac{1}{\beta^{2}} \sin ^{2} F\right) F^{\prime \prime}+\left(2 r F^{\prime}-\sin 2 F\right)- \\
-\frac{1}{\beta^{2}}\left(r F^{\prime 3}-F^{\prime 2} \sin 2 F+3 \frac{1}{r} F^{\prime} \sin ^{2} F-\frac{1}{r^{2}} \sin 2 F \sin ^{2} F\right)=0 .
\end{gathered}
$$

The next aim of our investigation is finding the solutions of equation (9).

Equation (9) is a very complicated nonlinear differential equation. In order to solve it, only numerical or approximation methods seem applicable. The crucial point of such analysis is that the leading derivative term in this equation contains the factor

$$
\Phi[F](r)=\left(r^{2}-\frac{1}{\beta^{2}} \sin ^{2} F\right) .
$$

If there exits $r_{0} \neq 0$ such that $F\left(r_{0}\right)= \pm \arcsin \left(\beta r_{0}\right)$, then it is easily shown that $\left.F^{\prime}(r)\right|_{r=r_{0}}=$ 0 . But if $r_{0}=0$, then $F(0)=\pi N$ where $N \in \mathbf{Z}$ and $\left.F^{\prime}(r)\right|_{r=0}=a \neq 0$. Using the standard procedure, one obtains the asymptotic behavior near this point $(r=0, F(0)=\pi N)$

$$
F(r)=\pi N+a r-\frac{7 a^{2}-4 \beta^{2}}{30\left(a^{2}-\beta^{2}\right)} a^{3} r^{3}+\underline{O}\left(r^{5}\right),
$$

where $a^{2}<\beta^{2} / 3$ is a constant. Of course, the derivative at the point $r=R$ is not zero.

To guarantee the stability of our solutions we should choose the following asymptotics at infinity $(r \rightarrow \infty)$

$$
F(r)=b(1 / r)^{2}-\frac{b^{3}}{21}(1 / r)^{6}-\frac{b^{3}}{3 \beta^{2}}(1 / r)^{8}+\underline{O}\left(1 / r^{10}\right),
$$




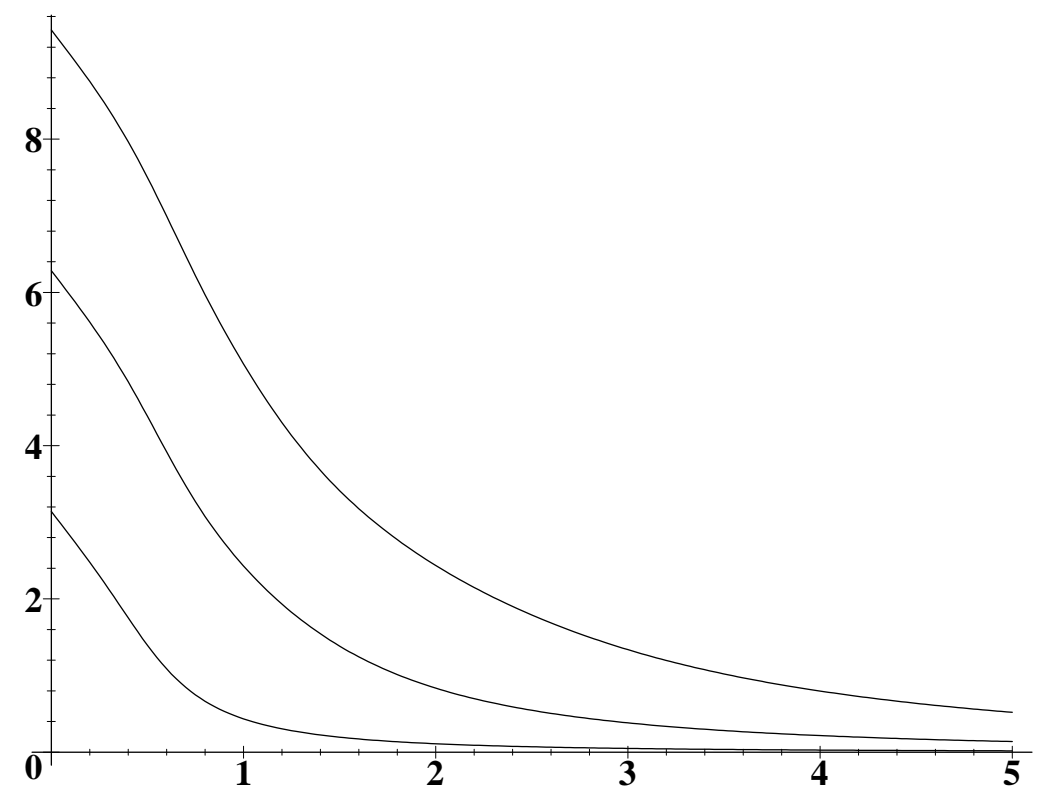

Figure 2: Solitons with $B=1,2$ and 3. Horizontal axis: $\mathrm{r}$ (in fm).

where $b$ is a constant. Solutions with such asymptotics at infinity and with asymptotics (11) $(N \neq 0)$ are stable because vacuum states $F(0)=\pi N$ and $F(\infty)=0$ are different.

Notice that equation (9) has very useful symmetries. First of all, this equation is symmetrical with respect to the changes $F \leftrightarrow F+N \pi, N \in \mathbf{Z}$ and $F \leftrightarrow-F$.

\begin{tabular}{|c|c||c|c|}
\hline $\begin{array}{c}\text { Baryon Charge, } \\
B\end{array}$ & $\begin{array}{c}\text { Energy of Soliton, } \\
E(\beta=1)\end{array}$ & $\begin{array}{c}\text { Baryon Charge, } \\
B\end{array}$ & $\begin{array}{c}\text { Energy of Soliton, } \\
E(\beta=1)\end{array}$ \\
\hline 1 & 3.487 & 6 & 300.08 \\
\hline 2 & 17.069 & 7 & 459.88 \\
\hline 3 & 47.150 & 8 & 668.05 \\
\hline 4 & 100.10 & 9 & 930.97 \\
\hline 5 & 182.28 & 10 & 1255.01 \\
\hline
\end{tabular}

Table 1: Dependence of the energy $E(B, \beta=1)$ from value of Baryon Charge $B$

The numerical investigation of the solutions of equation (9) which have the asymptotics (12) $(b>0)$ at infinity is presented in Fig.1. Most of such solutions can be evaluated only for $r>r_{0}$, where $r_{0}$ is determined by $F\left(r_{0}\right)= \pm \arcsin \left(\beta r_{0}\right)$. But among this set of solutions there are solutions with $r_{0}=0$. Such solutions have the asymptotics (11) at origin, and these are the topological solitons of the ChBI theory. The topological charge is defined by integral (21) as usual. Solitons with $B=1,2$ and 3 are presented in Fig.2. The scale parameter $\beta=807 \mathrm{MeV}$ is defined from the hypothesis that the soliton with $B=1$ is a nucleon. Indeed, 
using now a scale transformation in (7), one gets

$$
\beta=\frac{8 \pi f_{\pi}^{2}}{m_{p}} E(B=1, \beta=1)=807 \mathrm{MeV},
$$

where $E(B=1, \beta=1)=3.487$ is the energy of this soliton solution for $B=1$ and $\beta=1$.

In conclusion of this section let us consider the question about the asymptotics of energy of spherically symmetrical solitons at $B \rightarrow \infty$. In case of the Skyrme Model $E(B) \sim B^{2}[13$. In the Chiral-Born-Infeld's case it is not so. In Table 1 the values of the energy $E(B, \beta=1)$ for $B=1 \ldots 10$ are presented.

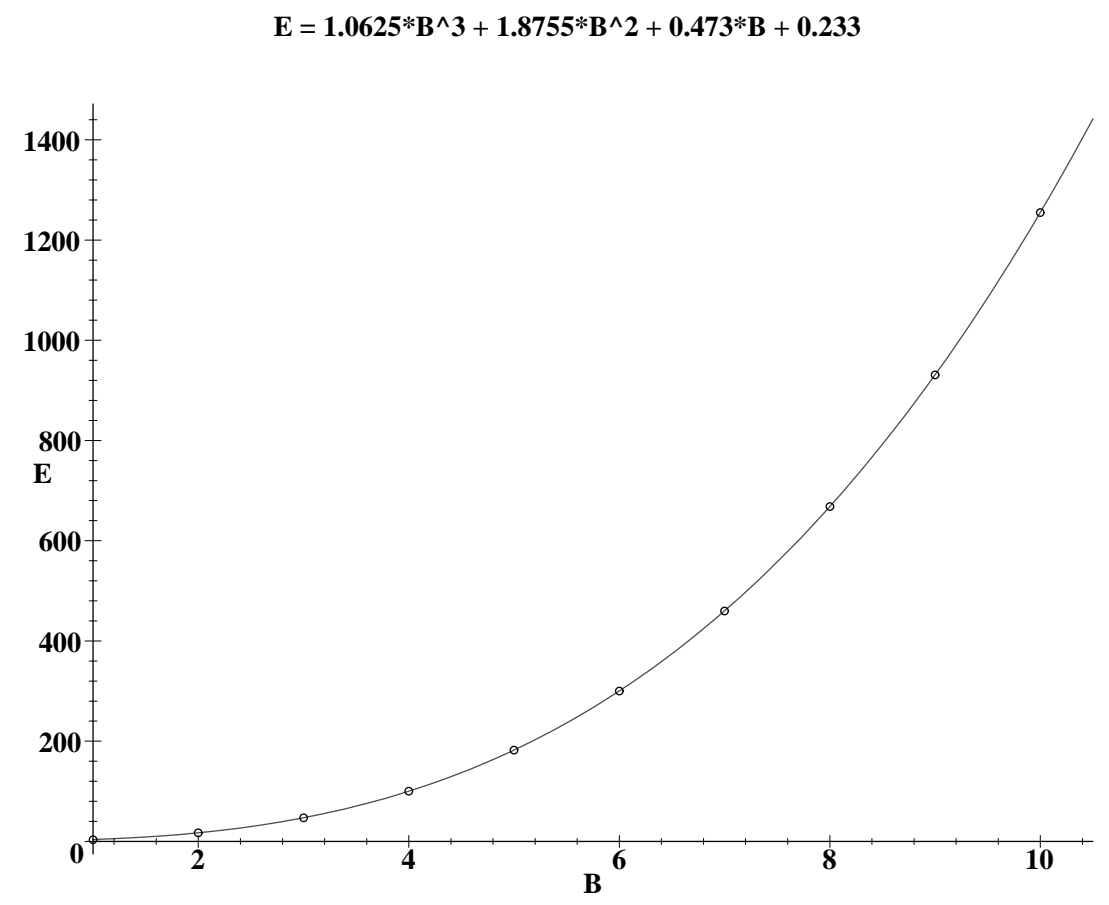

Figure 3: The energy $E(B, \beta=1)$ : interpolation of data of Table 1 .

It is easily shown that the Table 1 data can be interpolated by the expression

$$
E(B, \beta=1) \simeq 1.0625 B^{3}+1.8755 B^{2}+0.473 B+0.233 .
$$

This interpolation is shown in Fig. 3 where dots are the data from Table 1.

\section{Conclusions}

The aim of this paper is to construct a Born-Infeld theory for chiral fields. This theory has no singular solution and so the Chiral Born-Infeld theory is a good candidate on the role of effective chiral theory. In our work we show that this theory has the stable cluster finiteenergy solution. In the concept of a unified theory of mesons and baryons [1] such solutions are treated as the baryon states.

Of course, we do not give a comprehensive investigation of the Born-Infeld theory. The questions about non-spherical solutions, quantum fluctuations about such solutions and corresponding properties of baryons or about the nucleon-nucleon interaction are not clear now. 
But maybe the most important question in such investigation is about physical substantiation of such theory. All of these questions should be the themes for a future investigation.

In conclusion I would like to draw attantion to another class of solutions that were studied in Section 2. These solutions are defined everywhere, except the little $(\sim 0.2 \mathrm{fm})$ spherical region about the origin. These solutions look like a "bubble" of vacuum in the chiral fields and are of interest for the chiral bag model of baryons [14]. This question should be the theme for a future investigation too.

\section{References}

[1] T. H. Skyrme, Proc. Roy. Soc. Lond. A 260 (1961) 127; 230 (1955) 277.

[2] G. 't Hooft, Nucl. Phys. B 72 (1974) 461; G. S. Adkins, C. R. Nappi and E. Witten, Nucl. Phys. B 228 (1983) 552.

[3] I. Zahed and G. E. Brown, Phys. Rept. 142 (1986) 1; V. A. Nikolaev, Fiz. Elem. Chast. Atom. Yadra 20 (1989) 401.

[4] G. H. Derrick, J.Math.Phys. 5, 1252 (1964).

[5] B. L. Ioffe, Physics-Uspekhi No 12, (2002) 1273.

[6] G. S. Adkins and C. R. Nappi, Phys. Lett. B 137 (1984) 251; S. Yoro and T. Tatsumi, Prog.Theor.Phys. 78 (1987) 1392; D. Klabucar and G. E. Brown, Nucl. Phys. A 454 (1986) 589.

[7] S. Deser, M. J. Duff and C. J. Isham, Nucl. Phys. B 114 (1976) 29.

[8] J. Neto, C. Neves, E. R. de Oliveira and W. Oliveira, J. Phys. A 34 (2001) 5117.

[9] A. M. Polyakov and A. A. Belavin, JETP Lett. 22 (1975) 245 [Pisma Zh. Eksp. Teor. Fiz. 22 (1975) 503].

[10] B. Dion, L. Marleau and G. Simon, Phys. Rev. D 53 (1996) 1542.

[11] M. Born and L. Infeld, Proc. Roy. Soc. Lond. A 144 (1934) 425.

[12] N. K. Pak and C. H. Tze, Annals Phys. 117 (1979) 164; A. A. Tseytlin, Nucl. Phys. B 501 (1997) 41; D. Gal'tsov and R. Kerner, Phys. Rev. Lett. 84 (2000) 5955.

[13] E. B. Bogomolny and V. A. Fateev, Yad. Fiz. 37 (1983) 228.

[14] A. Hosaka and H. Toki, Phys. Rept. 277 (1996) 65. 